\title{
Tomographic PIV of flow through ordered thin porous media
}

\author{
I. A. Sofia Larsson ${ }^{1} \odot$ T. Staffan Lundström ${ }^{1} \cdot$ Henrik Lycksam ${ }^{1}$
}

Received: 9 January 2018 / Revised: 27 March 2018 / Accepted: 30 April 2018 / Published online: 16 May 2018

(c) The Author(s) 2018

\begin{abstract}
Pressure-driven flow in a model of a thin porous medium is investigated using tomographic particle image velocimetry. The solid parts of the porous medium have the shape of vertical cylinders placed on equal interspatial distance from each other. The array of cylinders is confined between two parallel plates, meaning that the permeability is a function of the diameter and height of the cylinders, as well as their interspatial distance. Refractive index matching is applied to enable measurements without optical distortion and a dummy cell is used for the calibration of the measurements. The results reveal that the averaged flow field changes substantially as Reynolds number increases, and that the wakes formed downstream the cylinders contain complex, three-dimensional vortex structures hard to visualize with only planar measurements. An interesting observation is that the time-averaged velocity maximum changes position as Reynolds number increases. For low Reynolds number flow, the maximum is in the middle of the channel, while, for the higher Reynolds numbers investigated, two maxima appear closer to each bounding lower and upper wall.
\end{abstract}

\section{Introduction}

Pressure-driven flow within the plane of a confined thin porous medium (TPM) takes place in a number of natural and industrial processes. This includes flow during manufacturing of fibre-reinforced polymer composites with liquid moulding processes (Frishfelds et al 2011; Nordlund and Lundström 2008; Tan and Pillai 2012), passive mixing in microfluidic systems (Jeon and Shin 2009), and paper making (Lundström et al 2002; Singh et al 2015).

The TPM considered here is a simplified, well-structured model of a porous medium where the solid parts have the shape of vertical cylinders placed on equal interspatial distance from each other in a quadratic pattern. The array of cylinders is placed between two parallel plates, and hence, the permeability of this quadratic array depends generally on both the diameter and height of the cylinders, as well as their interspatial distance (Fabricius et al 2016).

Depending on the geometrical ratios, three types of TPM can be distinguished (Fabricius et al 2016). Very thin porous medium (VTPM) where the cylinder height is much smaller than the interspatial distance, proportionally thin porous

\footnotetext{
I. A. Sofia Larsson

sofia.larsson@1tu.se

1 Division of Fluid and Experimental Mechanics, Luleå University of Technology, SE-97187 Luleå, Sweden
}

medium (PTPM) where the cylinder height is proportional to the interspatial distance, and homogeneously thin porous medium (HTPM) where the cylinder height is much larger than the interspatial distance.

Experiments are here performed on a model of a PTPM. In a previous study of the modeling of Darcian flow, Fabricius et al. (2016) found that, in this particular type of thin porous medium, three-dimensional effects in the flow exist and need to be taken into consideration when modeling the flow, since a two-dimensional assumption is no longer valid. Hence, it is suitable for further investigation with tomographic three-dimensional, three-component particle image velocimetry (3D3C PIV) which provides a detailed description of the flow field in only one measurement. The use of an experimental method also enables the study of higher Reynolds number flows.

\section{Experimental arrangements}

The experimental cell is made of PMMA (polymethyl methacrylate) with the internal dimensions $850 \times 200 \times 30 \mathrm{~mm}^{3}$. In a similar fashion as in Khayamyan et al $(2017 \mathrm{a}, \mathrm{b})$, it contains flow guiding vanes, a mesh with $2 \times 2 \mathrm{~mm}^{2}$ square openings, and a honeycomb with $4 \mathrm{~mm}$ circular holes to straighten the flow and promote uniform inlet flow to the porous domain. A valve, being located close to the outlet 
of the experimental cell and connected to a tube, is used to remove air bubbles entrained in the working fluid. This is necessary, since bubbles in the flow will influence the measurements and the results. The porous medium is modeled with cylindrical rods made of quartz glass with a diameter of $15 \mathrm{~mm}$ and a length of $35 \mathrm{~mm}$. Sixty rods are ordered in a structured (squared) array, six along the width, and ten along the length of the experimental cell. The rods are drilled 5 $\mathrm{mm}$ into the bottom of the experimental cell to remain stable when fluid flows through the domain, and extends all the way $(30 \mathrm{~mm})$ to the upper wall of the cell. The bed porosity is 0.8 , and hence, a relatively sparse system is studied. To investigate the effects from the entry conditions and the side walls on the flow and decide on the number of rods necessary, initial simulations based on the work described in Fabricius et al (2016) were done to aid the experimental design. The present setup ensures that no effects on the flow from the side walls are present in the measurement volume located in the middle of the array; the flow is also fully developed from free to porous flow and independent of entry conditions. Figure 1 shows an illustration of the
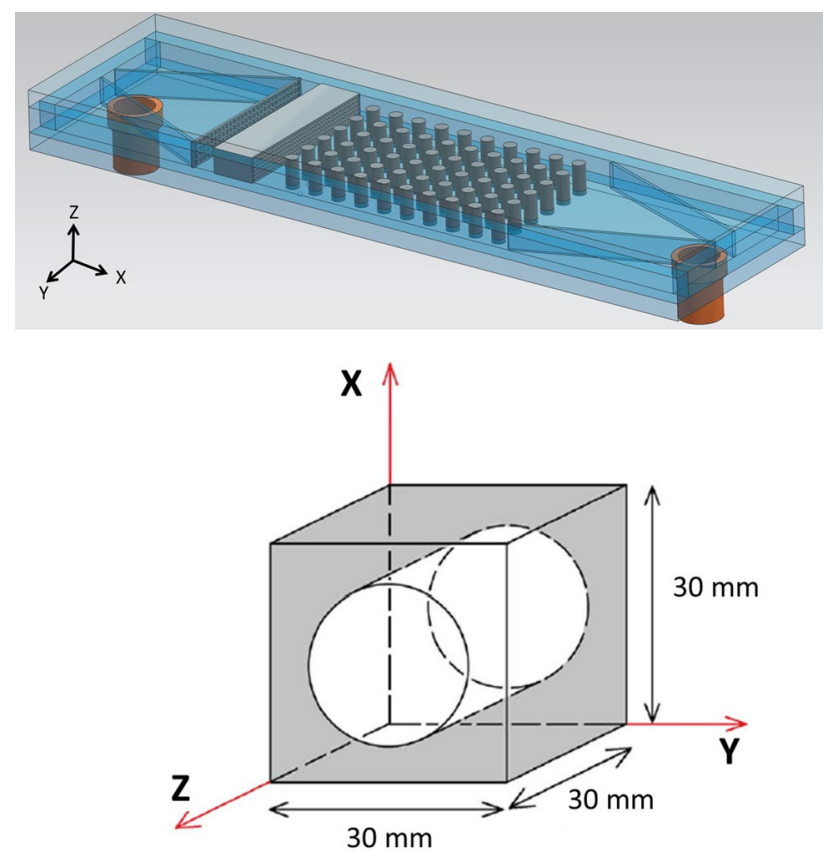

Fig. 1 Experimental cell with coordinate system and schematic of unit cell in the porous domain experimental cell, as well as a schematic of a unit cell in the porous domain.

\subsection{Refractive index matching}

To be able to accurately measure through the quartz glass cylinders without optical distortion, it is necessary to match the refractive index of the working fluid to that of the quartz cylinders. This can be done using two mixable fluids, one with a higher refractive index than quartz glass and one with a lower. Another criteria for these two fluids are that the combined kinematic viscosity $(v)$ should be as low as possible (to reduce the flow rate and pressure build-up inside the experimental cell) which excluded a glycerol-water mixture from consideration. Quartz glass has a refractive index $(n)$ of 1.4584 at a wavelength $(\lambda)$ of $589 \mathrm{~nm}$ and, as can be seen in Table 1, mineral oil and heptane (industrial petrol) fulfill the criteria above while still providing a relatively safe working environment. The dynamic viscosity $(\mu)$ of the working fluid was measured by a rheometer (Bohlin CVO, Malvern Instruments) and the density $(\rho)$ was measured through a Coriolis mass flow meter (MASSFLO flowmeter MASS 6000, Danfoss), and hence, the kinematic viscosity could be determined. A pump was driving the flow and a cooling system in the tank kept the fluid temperature constant at $25 \pm 0.1^{\circ} \mathrm{C}$ during the experiments.

When performing a refractive index matching, it is important to keep in mind that different substances have different chromatic dispersion, and therefore, it is not possible to achieve perfect refractive index matching between the quartz and working fluid for all wavelengths (at a given temperature). In the measurements, fluorescent particles, PMMA spheres dyed with rhodamine B from MicroParticles $\mathrm{GmbH}$ (diameter between $20-50 \mu \mathrm{m}$; density of 1.19 $\mathrm{g} / \mathrm{cm}^{3}$ ), were used as seeding. The emission spectra for these particles have a peak at $560 \mathrm{~nm}$ with a full width at half maximum (FWHM) of about $45 \mathrm{~nm}$ (all wavelengths below $540 \mathrm{~nm}$ are blocked by camera filters). Ideally, the refractive index matching should, therefore, be performed at the central peak wavelength of $560 \mathrm{~nm}$. Fortunately, all substances involved (oil, heptane, and quartz) exhibit normal chromatic dispersion $(d n / d \lambda<0)$ in the region around $560 \mathrm{~nm}$, and therefore, the error that arises from matching at another wavelength tends to partially cancel out. In the
Table 1 Fluid properties (for mineral oil and heptane, the specifications from the manufacturer are stated)

\begin{tabular}{llll}
\hline Fluid & $n$ & $v\left(\mathrm{~mm}^{2} / \mathrm{s}\right)$ & $\rho\left(\mathrm{kg} / \mathrm{m}^{3}\right)$ \\
\hline Mineral oil & $1.462-1.473$ at $20^{\circ} \mathrm{C}, 589 \mathrm{~nm}$ & $33-42$ at $20^{\circ} \mathrm{C}, 14-19$ & $827-862$ at $15^{\circ} \mathrm{C}$ \\
Heptane & 1.389 at $20^{\circ} \mathrm{C}, 589 \mathrm{~nm}$ & at $40^{\circ} \mathrm{C}$ & \\
Working fluid & 1.458 at $22^{\circ} \mathrm{C}, 589 \mathrm{~nm}$ & 0.55 at $25^{\circ} \mathrm{C}$ & 694 at $15^{\circ} \mathrm{C}$ \\
\end{tabular}


wavelength region from 532 to $589 \mathrm{~nm}$, the magnitude of the error is always $<3 \times 10^{-4}$ regardless of mixing ratio.

Another issue is that since the mineral oil and heptane are fluids, their refractive indices have significant temperature dependence, implying that the refractive index of the working fluid will change with temperature $(d n / d T=$ $-(3.7-5.0) \times 10^{-4} /{ }^{\circ} \mathrm{C}$ depending on mixing ratio).

In this work, the refractive index matching was done with two different methods, first roughly using a refractometer and then fine-tuned through laser beam refraction measurements. First, an Abbé refractometer from Zeiss with dispersion compensating prisms was used to measure the absolute refractive index of the working fluid at 589 $\mathrm{nm}$ and at a temperature of $22{ }^{\circ} \mathrm{C}$ (room temperature). Changing the sample temperature would have required the addition of a constant temperature water controller which was not available. However, the refractive index of the working fluid at operating conditions $\left(560 \mathrm{~nm}, 25^{\circ} \mathrm{C}\right)$ is similar to the refractive index at measurement conditions $\left(589 \mathrm{~nm}, 22^{\circ} \mathrm{C}\right.$ ), to an accuracy of $<4 \times 10^{-4}$ (depending on working fluid mixing ratio).

The relative refractive index difference between the working fluid and quartz cylinders was then measured using a low power $532 \mathrm{~nm}$ continuous diode laser. A sample of the working fluid was extracted and placed in a cuvette together with a single quartz cylinder. The laser beam was traversed through the quartz cylinder, and its refraction for various distances from the cylinder center was observed and measured on a screen. For a screen distance of $2 \mathrm{~m}$, refractive index differences down to $<1 \times 10^{-3}$ can be detected.

The final mixing ratio of mineral oil and heptane was approximately 5.5/1. Table 1 summarizes the fluid properties and Fig. 2 shows the experimental cell partly filled with the working fluid.

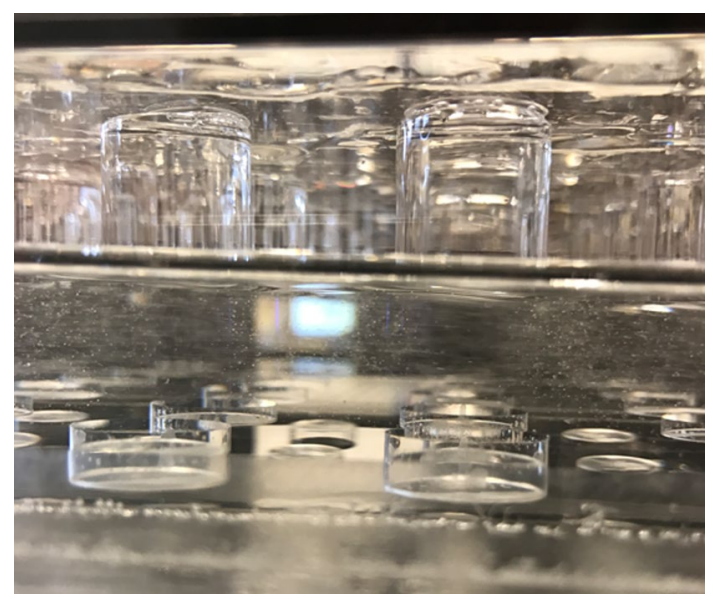

Fig. 2 Experimental cell partly filled with working fluid, note the tiny air bubbles entrained in the flow

\subsection{Tomographic PIV measurements}

The tomographic PIV system used is a commercially available system from Lavision GmbH. It consists of a doublepulsed Nd-YAG laser from Litron $(532 \mathrm{~nm}, 15 \mathrm{~Hz}, 200$ $\mathrm{mJ}$ ) with a laser guiding arm, four sCMOS cameras (5.5 MP, 16 bit, $6.5 \times 6.5 \mu \mathrm{m}$ pixel size), a programmable timing unit (PTU X) for triggering and synchronization, and a computer with the software Davis 8.4 to control the system and store the data. Volume optics generated the illumination. The cameras were mounted on a stable frame, viewing the experimental cell from above, and fitted with $100 \mathrm{~mm}$ $\mathrm{f} / 2.8$ macrolenses (the depth of field required $\mathrm{f}=11$ during the measurements) with longpass filters for fluorescent light $(540 \mathrm{~nm})$ to remove disturbing laser light reflections. The lenses were connected to Scheimpflug adapters and the laser light moved into the experimental cell from the side of it. Figure 3 shows an illustration of the experimental setup.

Accurate camera calibration is crucial for the results and the calibration can pose a challenge in a closed system like the one used in these experiments. Since there is no possibility to insert and remove a calibration target into the flow volume of interest, a dummy cell was built with the same dimensions but without the quartz glass cylinders. A calibration target was submerged in the dummy cell which was filled with the same working fluid as used in the real measurements. The real experimental cell was then temporarily replaced with the dummy cell and pictures were taken and the calibration could be performed. The design of the support of the cell ensured that the top surface of the cell was always kept at a fixed distance from the cameras. Since the same working fluid was used and refractive index matching applied, the camera calibration was also valid in the real measurement volume. Figure 4 shows the dummy cell used for calibration with the target inside.

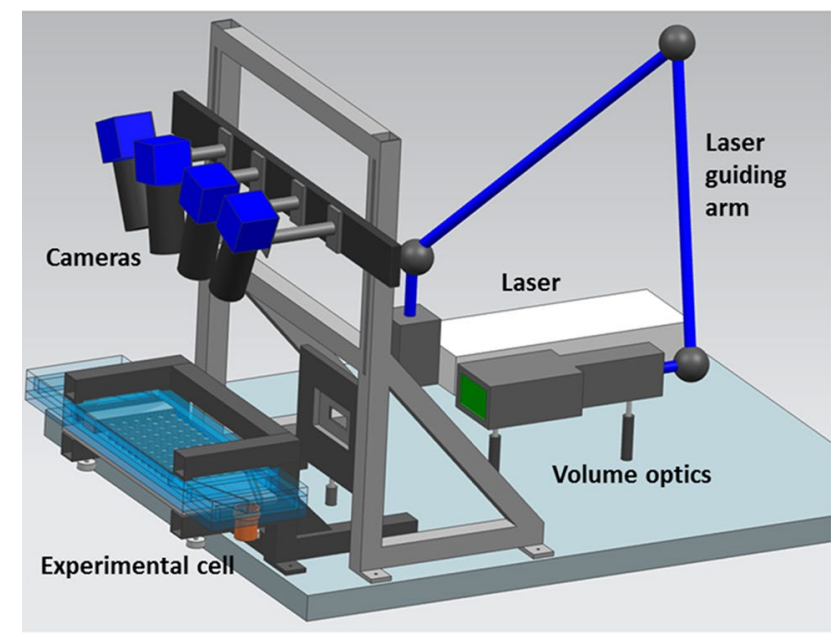

Fig. 3 Experimental setup 


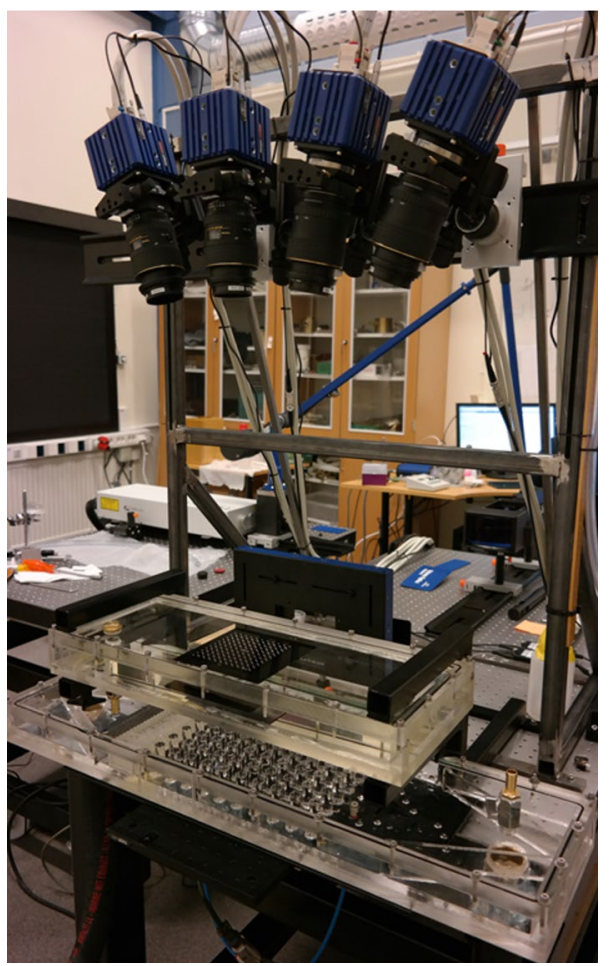

Fig. 4 Experimental cell replaced by dummy cell with calibration target inside

\subsubsection{Data acquisition}

The flow rates were varied to yield a particle Reynolds number $\left(\operatorname{Re}_{p}\right)$ of 45-950 based on the previous work by Khayamyan et al (2017a, b). Particle Reynolds number is commonly used for flow through porous media and is defined as $\operatorname{Re}_{p}=U_{\text {int }} D_{p} / \nu$, see Hlushkou and Tallarek (2006), for instance. In this equation, $D_{p}$ is the (average) particle diameter $U_{\text {int }}=U_{\text {Darcy }} / \phi$, where $\phi$ is bed porosity and $U_{\text {Darcy }}=Q / A_{\text {bed }}$, where $Q$ and $A_{\text {bed }}$ are volumetric flow rate and bed cross-sectional area, respectively. In this case, the bed corresponds to the array of cylinders where the cylinder diameter is used as the particle diameter. 250 image pairs were acquired at $10 \mathrm{~Hz}$ and the time between exposures was adjusted between 300 and $7000 \mu$ s depending on flow rate.

\subsubsection{Data processing and results}

The acquired images were preprocessed in several steps to ensure good reconstruction quality of the particles. The calibration procedure described earlier generated a mapping function between image and physical volume coordinates, and volume self-calibration (Wieneke 2008) was applied to correct and improve the mapping. The particle volume distribution was reconstructed using a fastMART (multiplicative algebraic reconstruction tomography) algorithm and the velocity vectors were calculated by iterative volume cross-correlation with a final interrogation volume size of $32 \times 32 \times 32$ voxel with $75 \%$ overlap. Spurious vectors were removed and replaced, the vector field smoothed $(3 \times 3 \times 3$ voxel), and finally, the areas in the resulting images containing no information were masked out. The final correlated volume had an extent of $80 \times 70 \times 30 \mathrm{~mm}^{3}$, containing $322 \times 271 \times 109$ velocity vectors. The scale factor was $29.3554 \mathrm{pixel} / \mathrm{mm}$ resulting in a vector spacing of approximately $0.27 \mathrm{~mm}$. In all graphs plotted, the velocity is scaled with the Darcian velocity, $U_{\text {Darcy }}$, and all results here presented are time-averaged.

Figure 5 shows time-averaged results from three different flow rates corresponding to a $\mathrm{Re}_{p}$ of 45,465 , and 950 , respectively. The flow rates represent close to Darcian flow, inertia flow, and turbulent flow. The flow direction is from left to right. Two perpendicular $(y z)$ planes cutting through the volume for respective case as well as one horizontal $(x y)$ plane in the middle of the measurement volume have been chosen to illustrate the flow field.

For $\mathrm{Re}_{p}=45$, the velocity plots resemble that in a rectangular channel both between the cylinders in the narrowest and the widest passage between them. Interestingly enough, this is not the case for the higher Reynolds numbers. For these cases, dog bone-shaped velocity contours develop and two velocity maxima appear. These shapes can be traced to separation of the flow from the cylinders, see, e.g., Van Dyke (1982). As $\operatorname{Re}_{p}$ increases, this will naturally happen first where the velocity is the highest, i.e., in the middle of the channel.

The velocity profiles along two lines in the $z$-direction, one in the middle of each perpendicular plane described above, can be seen in Fig. 6. The change in position of maximum velocity as $\operatorname{Re}_{p}$ increases, as already revealed in Fig. 5, is here quantified. It is also seen that the scaled velocity in these middle positions decreases with $\mathrm{Re}_{p}$, an effect due to continuity, since the velocity profile also becomes flatter in the $y$-direction (not explicitly shown here).

Figure 7 shows the streamlines of scaled velocity in the middle of the measurement volume and in one wake behind a cylinder. As appears, the flow field changes considerably when $\operatorname{Re}_{p}$ increases. The streamlines starting in the horizontal midplane have a wavy motion at low $\operatorname{Re}_{p}$ that is not seen for higher $\operatorname{Re}_{p}$, i.e., at low $\operatorname{Re}_{p}$, the main flow moves into the open spaces formed downstream each cylinder. At higher $\mathrm{Re}_{p}$, inertia becomes stronger, hindering the main flow to move into this space and relatively large wakes are formed. One of these wakes at each $\operatorname{Re}_{p}$ is illustrated. As $\mathrm{Re}_{p}$ increases, two attached vortices are formed in the wake. For $\operatorname{Re}_{p}=465$, it can be seen that when approaching the upper wall of the measurement volume, the streamlines from each vortex tilt towards each other and are no longer clearly separated as they are for the case with $\operatorname{Re}_{p}=950$. 

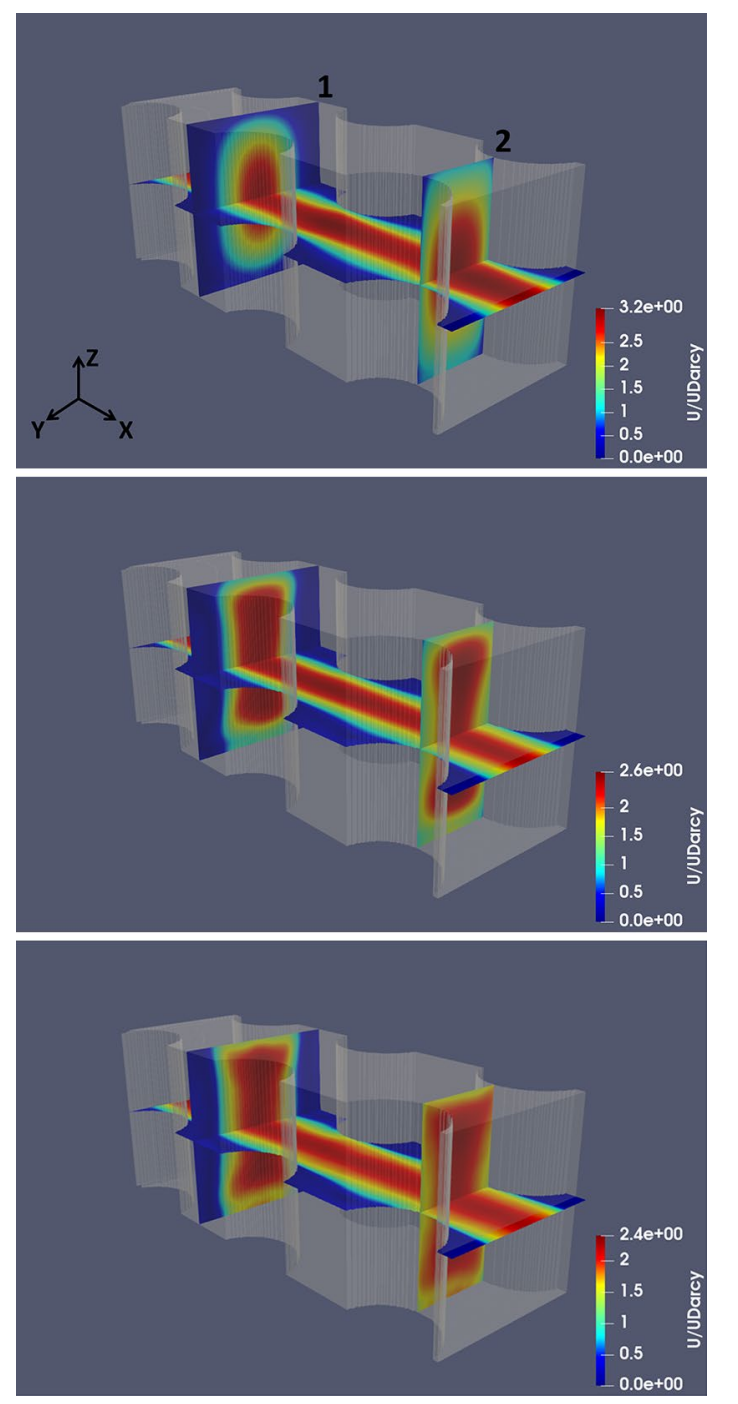

Fig. 5 Contour plots of time-averaged velocity, $\operatorname{Re}_{p}=45,465,950$, respectively, from top to bottom. Two perpendicular ( $y z$ ) planes, one located at the widest passage (labeled 1) and the other one between the cylinders at the narrowest passage (labeled 2). One horizontal ( $x y$ ) plane in the middle of the measurement volume. Flow from left to right in the positive $x$-direction

To further visualize this, a vortex identification parameter (the Q-criterion) is plotted in Fig. 8 for $\operatorname{Re}_{p}=465$, revealing highly three-dimensional complex vortex structures in the wake behind the cylinders.

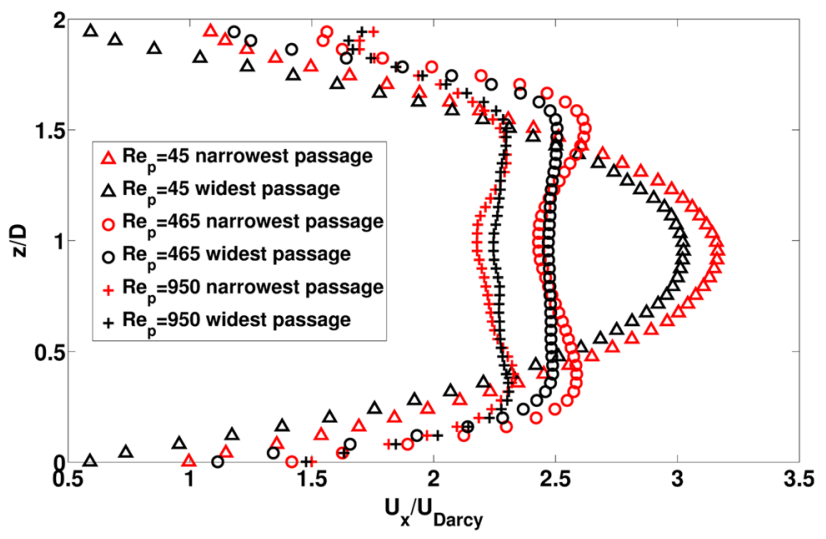

Fig. 6 Velocity profiles along two lines in the $z$-direction in two different perpendicular planes, one in the narrowest and one in the widest passage between the cylinders

\section{Conclusions}

The experiments demonstrate the possibility to measure the flow field in a model of a porous medium. The advantage of capturing all three velocity components in a volume becomes obvious already in this simplified, structured model where three-dimensional effects arise in the wakes behind the cylinders, especially when $\operatorname{Re}_{p}$ increases. Another main result from this first study is that the time-averaged velocity maximum changes position as $\mathrm{Re}_{p}$ increases. For low Reynolds number flow, the maximum is in the middle of the channel, while, for the higher Reynolds numbers investigated, two maxima appear closer to each bounding lower and upper wall. This will be further investigated and is probably due to separation of the flow from the cylinders in the middle of the channel.

From a practical point of view, three-dimensional measurements, such as these, enable detailed studies where different general flow phenomena can be coupled to integrated quantities such as permeability and dispersion, and porous media can easier than before be tailor-made for different purposes and applications.

The refractive index matching works well and offers a relatively safe working environment and a simple way to perform PIV measurements without optical distortion. The results will be further analyzed and the experimental 

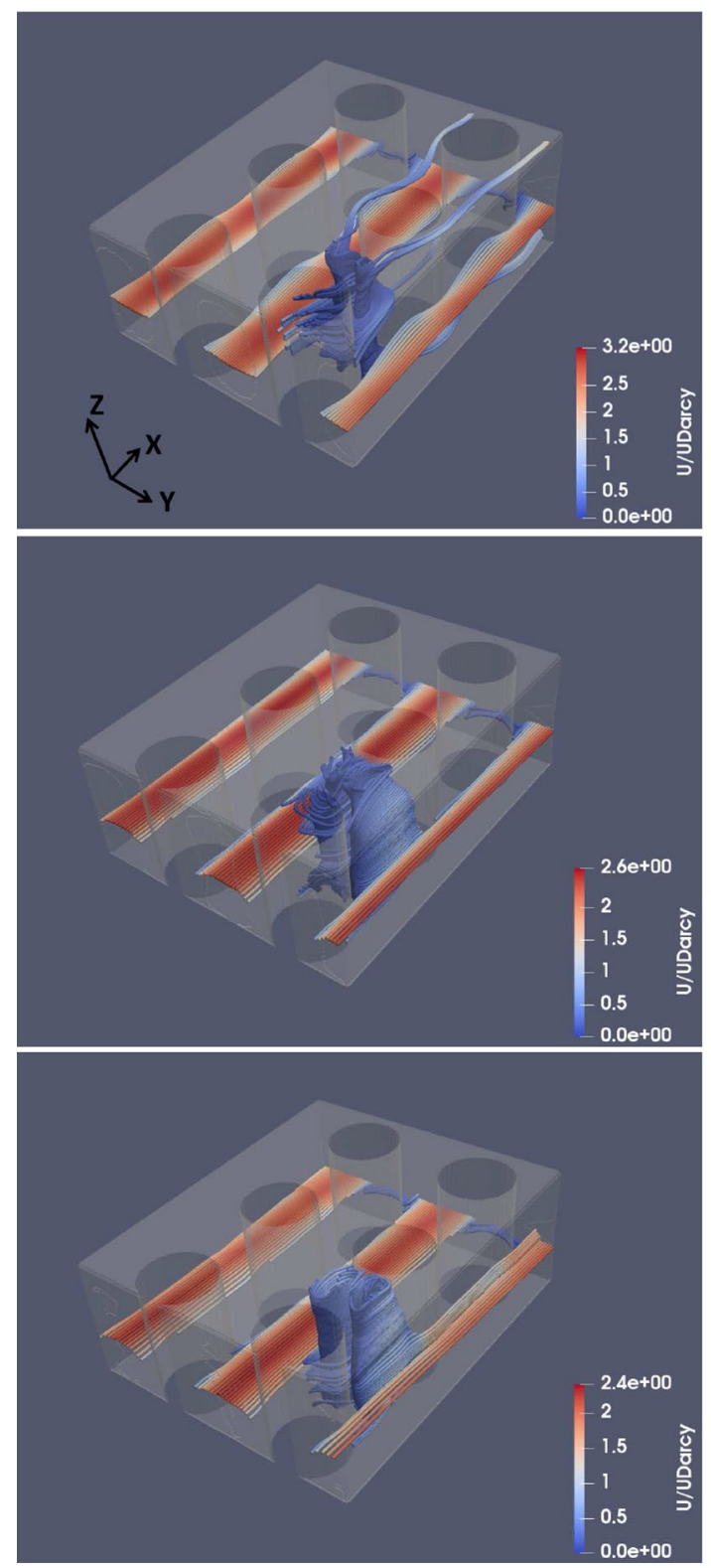

Fig. 7 Results from the measurements showing streamlines in the volume, $\operatorname{Re}_{p}=45,465,950$ from top to bottom, time-averaged. Flow from left to right in the positive $x$-direction

methodology will be used in future tomographic PIV measurements of flow through models of porous materials, with different types of structures, to provide a deeper understanding of the flow dynamics.

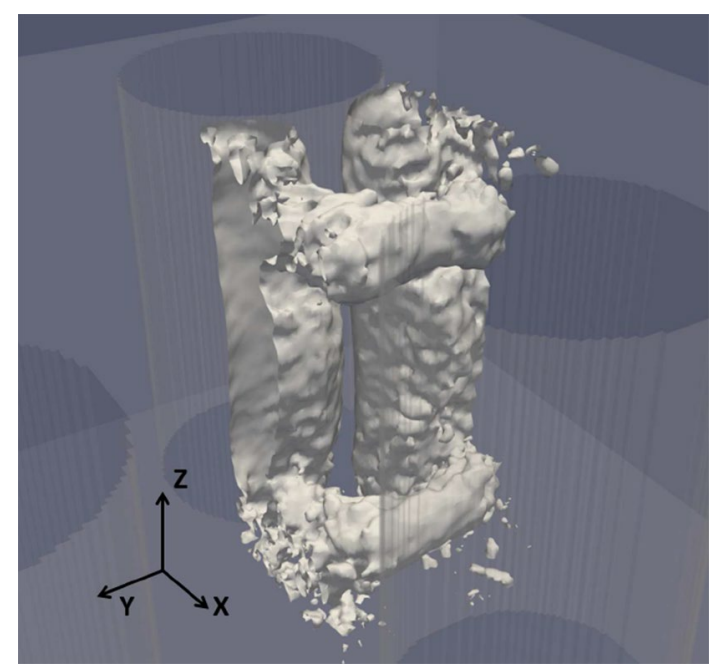

Fig. 8 Visualization of the vortex structure in the wake behind a cylinder for $\operatorname{Re}_{p}=465$. Isocontours of Q-criterion in a solid color to enhance the visibility of the horseshoe-like structures. Flow from left to right in the positive $x$-direction

Acknowledgements The equipment was funded by the Kempe Foundations and Luleå University of Technology, and the work was carried out under the grant 2017-04390 from the Swedish Research Council and within the framework of the VINNOVA VINN Excellence Centre DRIZZLE.

Open Access This article is distributed under the terms of the Creative Commons Attribution 4.0 International License (http://creativeco mmons.org/licenses/by/4.0/), which permits unrestricted use, distribution, and reproduction in any medium, provided you give appropriate credit to the original author(s) and the source, provide a link to the Creative Commons license, and indicate if changes were made.

\section{References}

Fabricius J, Hellström JGI, Lundström TS, Miroshnikova E, Wall P (2016) Darcys law for flow in a periodic thin porous medium confined between two parallel plates. Transp Porous Med 115(3):473-493

Frishfelds V, Lundström TS, Jakovics A (2011) Lattice gas analysis of liquid front in non-crimp fabrics. Transp Porous Med 84:75-93

Hlushkou D, Tallarek U (2006) Transition from creeping via viscous-inertial to turbulent flow in fixed beds. J Chromatogr 1126(1-2):70-85

Jeon W, Shin CB (2009) Design and simulation of passive mixing in microfluidic systems with geometric variations. Chem Eng J 152:575-582

Khayamyan S, Lundström TS, Gren P, Lycksam H, Hellström JGI (2017a) Transitional and turbulent flow in a bed of spheres as measured with stereoscopic particle image velocimetry. Transp Porous Med 117(1):45-67

Khayamyan S, Lundström TS, Hellström JGI, Gren P, Lycksam H (2017b) Measurements of transitional and turbulent flow in a randomly packed bed of spheres with particle image velocimetry. Transp Porous Med 116(1):413-431 
Lundström TS, Toll S, Hakanson JM (2002) Measurements of the permeability tensor of compressed fibre beds. Transp Porous Med 47:363-380

Nordlund M, Lundström TS (2008) Effect of multi-scale porosity in local permeability modelling of non-crimp fabrics. Transp Porous Med 73:109-124

Singh F, Stoeber B, Green SI (2015) Micro-PIV measurement of flow upstream of papermaking forming fabrics. Transp Porous Med 107:435-448

Tan H, Pillai KM (2012) Multiscale modeling of unsaturated flow in dual-scale fiber preforms of liquid composite molding. isothermal flows. Compos Part A Appl Sci Manuf 43:1-13
Van Dyke M (1982) An Album of Fluid Motion. The Parabolic Press, Stanford

Wieneke B (2008) Volume self-calibration for 3D particle image velocimetry. Exp Fluids 45:549-556

Publisher's Note Springer Nature remains neutral with regard to jurisdictional claims in published maps and institutional affiliations. 